\title{
A HISTÓRIA DA MATEMÁTICA NO ENSINO E NA APRENDIZAGEM DA MULTIPLICAÇÃO
}

\section{THE HISTORY OF MATHEMATICS IN TEACHING AND LEARNING MULTIPLICATION}

\author{
Adriana Borges de Paiva ${ }^{1}$ \\ Universidade Federal de Uberlândia
}

\begin{abstract}
Resumo
O presente trabalho apresenta resultados parciais de uma pesquisa de natureza bibliográfica que estudou a importância da utilização da História da Matemática como recurso metodológico no desenvolvimento da prática pedagógica nos primeiros anos do Ensino Fundamental. No texto são enfatizadas questões vinculadas ao ensino dos algoritmos da multiplicação. Também são apresentados e descritos vários procedimentos, técnicas e métodos produzidos e utilizados no passado, por diversos povos, em diferentes regiões, para a realização de cálculos relacionados a multiplicação, a saber: a multiplicação realizada com os dedos das mãos, a multiplicação desenvolvida pelos egípcios, a multiplicação em gelosia ou multiplicação em grade, a multiplicação utilizada pelos russos e a multiplicação pelas barras criadas pelo escocês John Napier. O texto apresenta ainda ideias importantes que justificam a utilização da História da Matemática no processo educativo e os impactos que promove na aquisição de conhecimentos por parte do aprendiz. Pelo estudo realizado foi constatado que o recurso à História da Matemática como estratégia metodológica contribui efetivamente para a melhoria do ensino e da aprendizagem dos conteúdos matemáticos trabalhados pelos docentes nos anos iniciais. A História da Matemática motiva e estimula a participação dos alunos no processo educativo, enriquece o desenvolvimento das aulas, esclarece dúvidas e questionamentos, demonstra a evolução dos conceitos e das ideias matemáticas ao longo do tempo, deixando claro que esta ciência está em constante evolução, em permanente transformação, rompendo assim com as ideias de alguns professores e alunos que concebem a Matemática como um saber estático, hermético, composto de conhecimentos inquestionáveis e imutáveis.
\end{abstract}

Palavras-chave: História da Matemática; Ensino e Aprendizagem; Multiplicação.

\begin{abstract}
\section{${ }^{1}$ adrianapaiva@ufu.br}

The present work presents results of a research of a bibliographic nature that studies the importance of the use of the History of Mathematics as a methodological resource in the development of pedagogical practice in the first years of Elementary School. Without text are the issues related to the teaching of multiplication algorithms. What is new in actions, the techniques and methods they produce and use in the past, in a world, in various regions, for a publication of a multiplication recipe, a saber: a multiplication done with the hands, a multiplicity developed by the an increase in lattice or multiplication in degree, a multiplication made by the Russians and multiplication by 
the bars created by the Scotsman John Napier. The text also presents important ideas that justify a use of the History of Mathematics in the educational process and those that promote the dissemination of knowledge by the learner. The study was carried out using the History of Mathematics as a strategy for the improvement of teaching and for the exercise of curricula in the initial years. The History of Master's Mathematics and Stimulating Killing Participation in the Educational Process, Ending Classroom Development, Closing Session and Questioning, Demonstrating the Evolution of Mathematics and Mathematical Ideas Over Time, in permanent transformation, thus breaking with ideas of some teachers and students who are Mathematics as a static, hermetic knowledge, composed of unquestionable and immutable knowledge.

Keywords: History of Mathematics; Teaching and learning; Multiplication.

\section{Introdução}

Nos anos iniciais do Ensino Fundamental, ao ensinar as operações básicas, os professores, de maneira geral, instruem os alunos para a aprendizagem do algoritmo da multiplicação da seguinte forma:

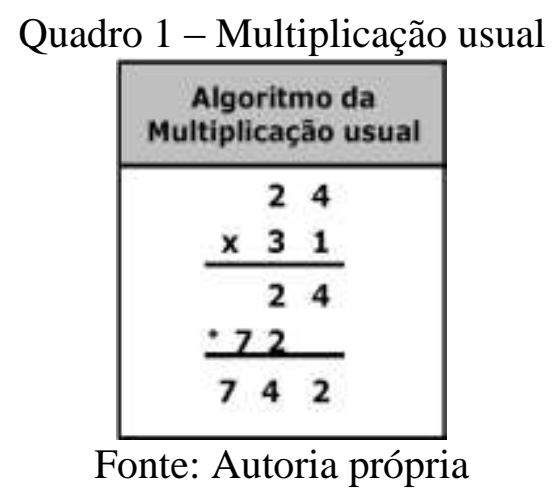

Essa forma de ensinar, acaba sendo, basicamente, no desenvolvimento da prática pedagógica, a única estratégia apresentada pelo professor, dando a entender muitas vezes que não existe outro algoritmo para desenvolver a multiplicação.

Na prática de sala de aula, para que o aluno domine esse algoritmo, prioriza-se a exercitação, o treino, visando a aquisição de automatismos que possibilitem a mera reprodução dos passos que o professor repassa, geralmente, por meio de transmissão oral e explicações dadas a partir de exemplos apresentados na lousa. Não há a preocupação com o desenvolvimento mais amplo do aluno, em termos intelectuais e culturais. A efetiva compreensão de como funciona a multiplicação e sua aplicabilidade 
é secundária, o importante é que aluno consiga fazer, repetir os passos que foram estabelecidos e instruídos pelo professor.

\begin{abstract}
A consequência dessa visão em sala de aula é a imposição autoritária do conhecimento matemático por um professor que, supõe-se, domina e o transmite a um aluno passivo, que deve se moldar à autoridade da "perfeição científica". Outra consequência e, talvez, a de resultados mais nefastos, é a de que o sucesso em matemática representa um critério avaliador da inteligência dos alunos, na medida em que uma ciência tão nobre perfeita só pode ser acessível a mentes privilegiadas, os conteúdos matemáticos são abstratos e nem todos têm condições de possuí-los (CARVALHO, 1994, p.15).
\end{abstract}

Essa forma de desenvolver o trabalho pedagógico, não estimula o pleno envolvimento do aluno no processo de aprender, não desperta o interesse em aprender, impactando na qualidade da aprendizagem, o que é comprovado pelo baixo rendimento apresentado pelos alunos dos anos iniciais, em questões presentes em exames, por exemplo a Prova Brasil, em que são exigidas formas de pensar e raciocinar relacionadas a multiplicação.

Para que o trabalho educativo se torne mais dinâmico e motivador, o professor poderá utilizar da História da Matemática no desenvolvimento da prática pedagógica. A História da Matemática, de acordo com Brasil (1997, p. 45) “[...] mediante um processo de transposição didática e juntamente com outros recursos didáticos e metodológicos, pode oferecer uma importante contribuição ao processo de ensino e aprendizagem em Matemática".

No caso do ensino da multiplicação, o professor poderá recorrer a vários exemplos históricos de como no passado os seres humanos desenvolviam esse procedimento de cálculo.

O trabalho pedagógico com outros algoritmos, utilizados por diferentes povos no passado, em diferentes regiões do mundo, valoriza a Matemática enquanto conhecimento social, envolve o aluno no processo formativo permitindo que ele faça comparações entre o processo de cálculo já conhecido, identificando diferenças e semelhanças, percebendo as vantagens e desvantagens de cada um dos procedimentos e estratégias de cálculo. Essa forma favorece uma melhor compreensão dos conteúdos estudados permitindo que, numa dada operação o aluno escolha o algoritmo que considere mais adequado, mais interessante enfim, aquele com que tenha mais afinidades. 
O professor, ao desenvolver ações educativas na sala de aula que estabelecem comparações entre saberes matemáticos produzidos e utilizados no passado com saberes matemáticos do tempo presente, de acordo com Brasil (1997, p. 34), "[...] tem a possibilidade de desenvolver atitudes e valores mais favoráveis do aluno diante do conhecimento matemático".

\title{
Alguns procedimentos de multiplicação utilizados no passado
}

Para Ifrah (1992), a mão do ser humano ao longo do tempo serviu para efetuar contagens e para desenvolver cálculos. Vestígios históricos encontrados, por exemplo, na índia, no Iraque, na Síria, no norte da África, indicam que o homem fazia uso das mãos para realizar multiplicações.

Referindo-se ao uso dos dedos das mãos para representação dos números, Eves (2011), afirma:

\begin{abstract}
Além dos números falados, numa certa época usaram-se largamente os números digitais (representados por meio de dedos). Com efeito, a expressão de números por meio de várias posições dos dedos e das mãos talvez preceda os símbolos numéricos ou os nomes dos números. Assim, os símbolos escritos primitivos para $1,2,3$ e 4 eram invariavelmente o número conveniente de riscos verticais ou horizontais, representando o número correspondente de dedos levantados ou estendidos, remontando a palavra digito (isto, e "dedo"), para indicar os algarismos de 1 a 9, a mesma origem (EVES, 2011, p. 29).
\end{abstract}

De acordo com o pensamento desenvolvido por Ifrah (1992) para realizar as multiplicações de todos os números compreendidos entre 5 e 10 agia-se da seguinte forma:

Para multiplicar, 7 por 8, por exemplo, utilizando os dedos das mãos, dobrava-se numa das mãos tantos dedos quantas unidades suplementares há em 7 com relação a 5 (isto é: $7-5=2$ dedos) e mantinha os outros três estendidos. Em seguida, dobrava-se na outra mão os dedos correspondentes às unidades suplementares de 8 em relação a 5 (isto é: $8-5=3$ dedos), mantendo os outros dois estendidos. $\mathrm{O}$ resultado era obtido multiplicando por 10 o número de dedos dobrados nas duas mãos, ou seja, $(2+3)$ x $10=$ 50, acrescentando em seguida a este resultado parcial ao produto dos dedos levantados da primeira mão pelos dedos levantados da outra mão, ou seja, $(3$ × $2=6)$. Assim, se chegava a: $7 \times 8=(2+3) \times 10+(3 \times 20)=56$

O quadro a seguir ilustra esse processo: 
Quadro 2 - Multiplicação de 7 por 8

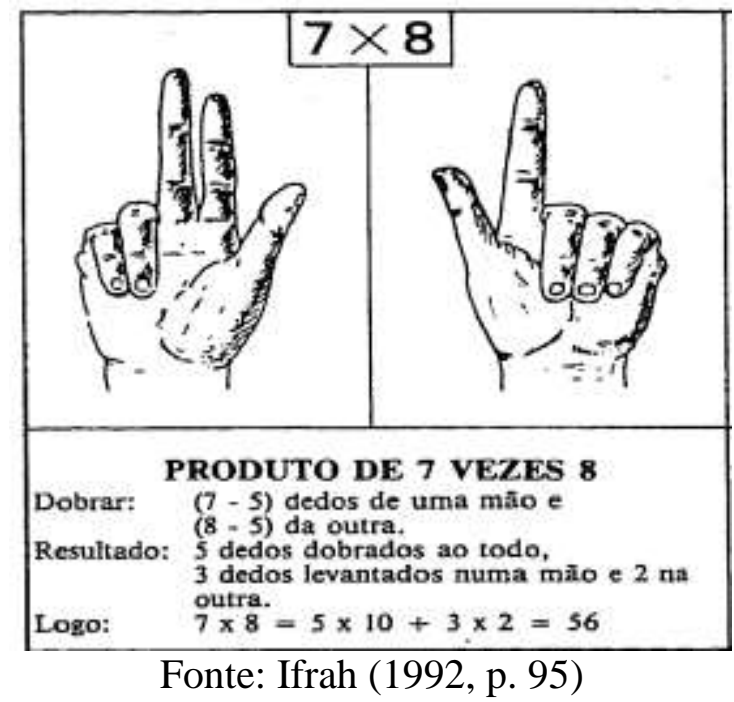

O quadro 3 apresenta outro exemplo:

Quadro 3 Multiplicação de 8 por 6

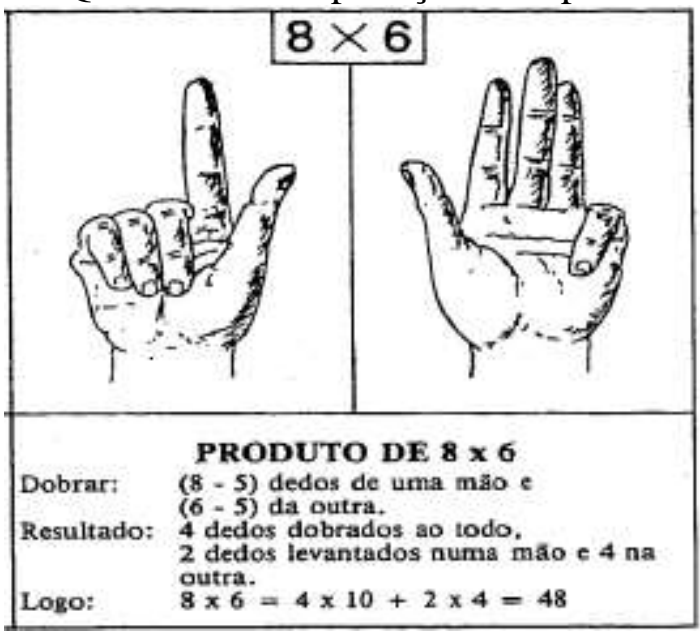

Fonte: Ifrah (1992, p. 95)

No Egito Antigo, de acordo com Eves (2011), as multiplicações eram obtidas através de dobras. Para realizar a multiplicação de 18 por 15, por exemplo, os egípcios procediam da seguinte maneira: 
$\left.1^{\circ}\right)$ Dobra-se sucessivamente o 15 :

$\begin{array}{lllcll}1 & 2 & 4 & 8 & 16 & \ldots \\ 15 & 30 & 60 & 120 & 240 & \ldots\end{array}$

$2^{\circ}$ ) Determinava-se o resultado de 18 x 15 da seguinte forma:

$18=\mathbf{2}+\mathbf{1 6}$

$18 \times 15=2 \times 15+16 \times 15$

$18 \times 15=30+240$

$18 \times 15=270$

Eves (2011, p. 72) apresenta o seguinte exemplo de multiplicação egípcia:

\begin{tabular}{|c|c|}
\hline \multicolumn{2}{|c|}{26 por 33} \\
\hline 1 & 33 \\
\hline 2 & 66 \\
\hline 4 & 132 \\
\hline 洋 8 & 264 \\
\hline 16 & 528 \\
\hline
\end{tabular}

Como $26=2+8+16$ (aqueles indicados com asteriscos), basta somarmos os múltiplos correspondentes de 33 (aqueles indicados com círculos), ou seja, $66+264+$ 528 e chega-se a resposta 858.

A multiplicação na Índia, conforme Boyer (1974), provavelmente a partir do século XII, era desenvolvida por meio de um procedimento que recebia vários nomes: multiplicação em reticulado, multiplicação em gelosia, ou em cédula ou em grade ou quadrilateral.

Boyer (1974, p. 158) afirma que esse procedimento da Índia foi levado para a China e à Arábia. "Dos árabes passou para a Itália nos séculos quatorze e quinze e lá o nome gelosia lhe foi associado por causa da semelhança com os gradeados colocados em frente as janelas em Veneza e outros lugares".

Para Eves (2011), a gelosia ou método da grade, 
Foi um dos métodos favoritos dos árabes, através dos quais passou para a Europa Ocidental. A simplicidade de sua aplicação o poderia te-lo mantido em uso ate hoje, não fora a necessidade de imprimir, ou desenhar, uma rede de segmentos de reta. O modelo lembra uma grade de janela chamada "gelosia" (em francês "jalousie" que significa "rotula") (EVES, 2011, p. $323)$.

Para obter o resultado da multiplicação de 32 por 24 usando a gelosia os povos antigos procediam da seguinte forma:

$\mathbf{1}^{\mathbf{0}}$ passo: Realizar multiplicações parciais.
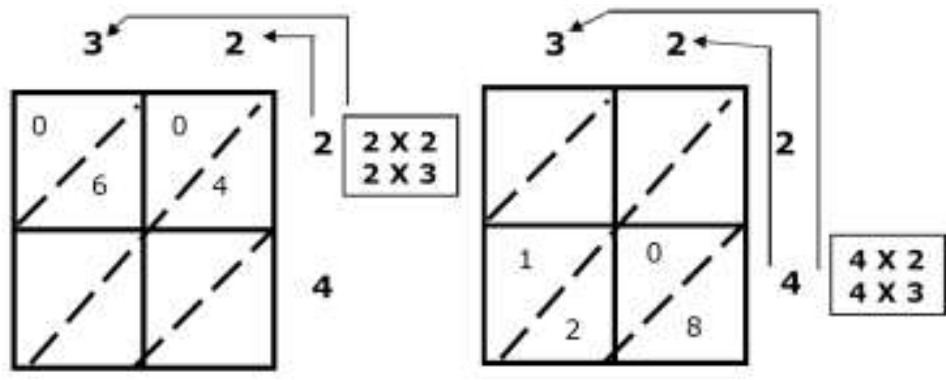

$2^{\mathbf{0}}$ passo: Realizadas as multiplicações parciais, para obter o produto eram somandos os valores que se encontram nas diagonais.

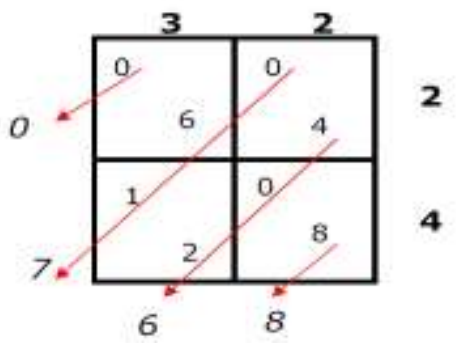

$32 \times 24=768$

Eves (2011, p. 254), apresenta o seguinte exemplo de multiplicação realizada pelo método da grade ou gelosia $(135$ x $12=1620)$ :

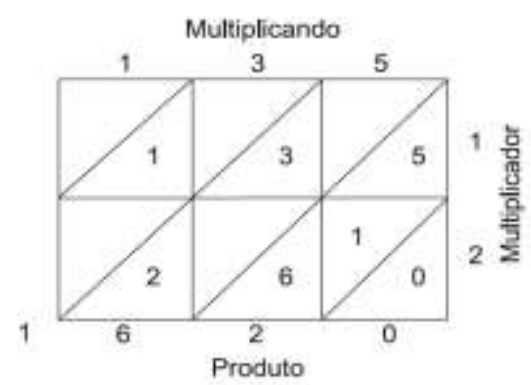


A multiplicação russa, de acordo com Souza (2001), era um procedimento de cálculo utilizado pelos camponeses russos para resolver multiplicações. Esta forma de multiplicar consistia em dividir por 2 de maneira sucessiva o primeiro fator da multiplicação e ao mesmo tempo ir dobrando o segundo fator. $\mathrm{O}$ resultado era obtido a partir da soma dos valores encontrados na dobra cuja correspondência na metade fosse ímpar.

Para calcular a multiplicação de 12 por 24, na prática procedia-se da seguinte maneira:

$1^{\mathrm{o}}$ Escrevia se os dois fatores, um ao lado do outro, formando duas colunas:

$12 \quad 24$

$2^{\circ}$ Determinava-se a metade do primeiro fator e o dobro do segundo, de forma sucessiva:

$\begin{array}{cc}12 & 24 \\ 6 & 48 \\ 3 & 96 \\ 1 & 192\end{array}$

$3^{\circ}$ Identifica-se na primeira coluna os números ímpares (no caso 3 e 1 ) e os números correspondentes na segunda coluna (no caso 96 e 192).

Para obter o resultado soma-se $96+192$. Portanto, 12 × $24=96+192=288$

Veja outro exemplo: 25 x 15

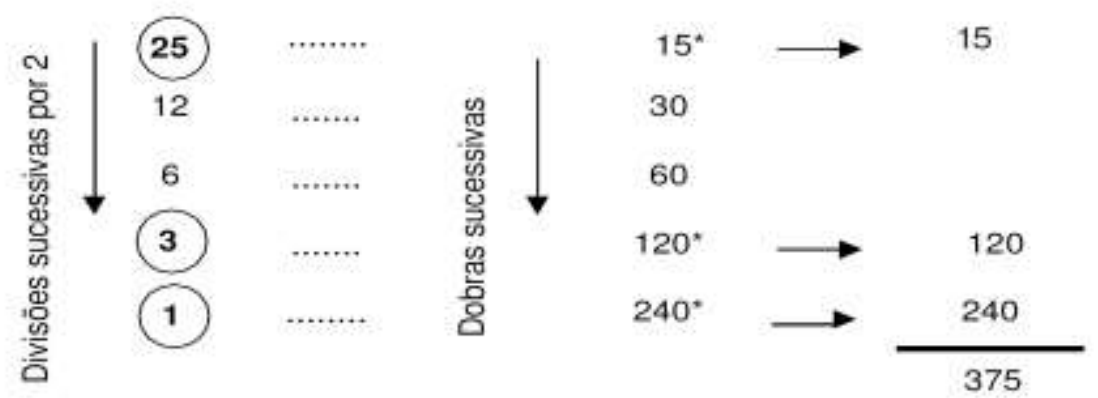

Portanto, $25 \times 15=15+120+240=375$

De acordo com Eves (2011), em 1617, o matemático Escocês, John Napier, criou uma técnica de multiplicação, conhecida como "barras de Napier" ou "ossos de Napier".

Eram tão amplas as dificuldades experimentadas na multiplicação de números grandes que se buscaram métodos mecânicos para levar a cabo o processo. Nesse sentido a invenção de Napier, conhecida como barras de 
Napier ou ossos de Napier, descrita em seu trabalho, Rabdologiae, publicado em 1617, conseguiu alcançar muita fama (EVES, 2011, p. 369).

As barras de Napier são constituídas de 10 tiras. Estas tiras como se observa na ilustração abaixo, são elaboradas e organizadas a partir de determinados números $(0,1$, $2,3,4,5,6,7,8,9)$ escritos nas partes superiores e seus respectivos múltiplos escritos nas partes inferiores.
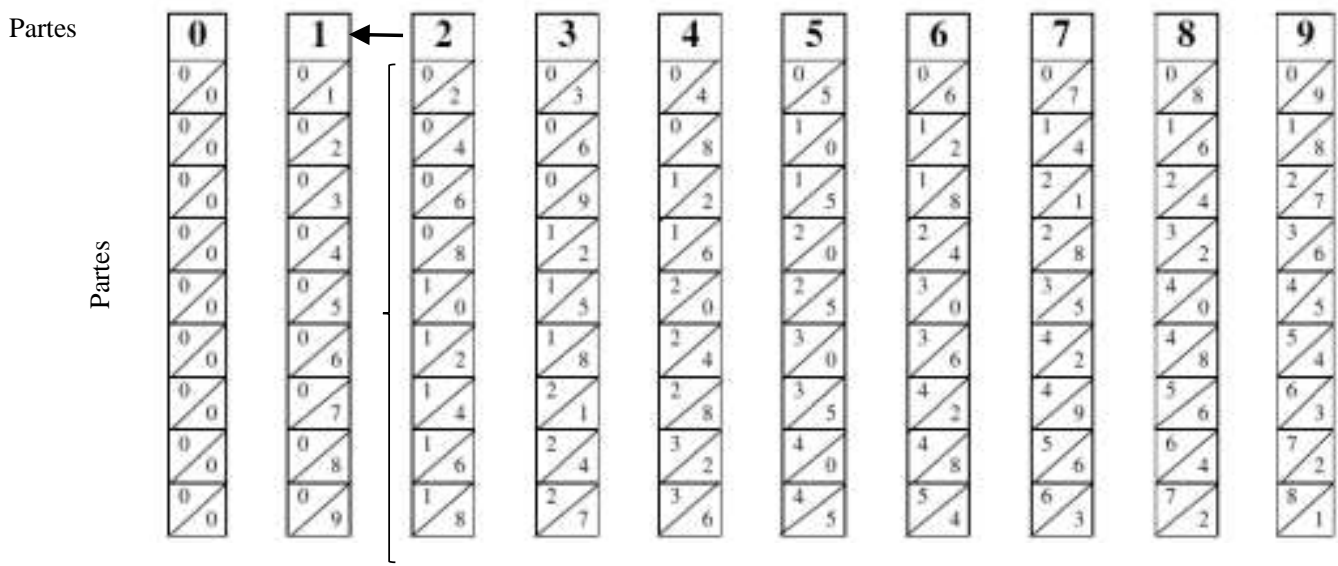

O desenvolvimento da multiplicação com as "barras de Napier" funciona da seguinte forma: Por exemplo, para multiplicar 35 por 24. Colocam-se as tiras encabeçadas por 3 e 5 lado a lado, tal como mostra a ilustração abaixo.

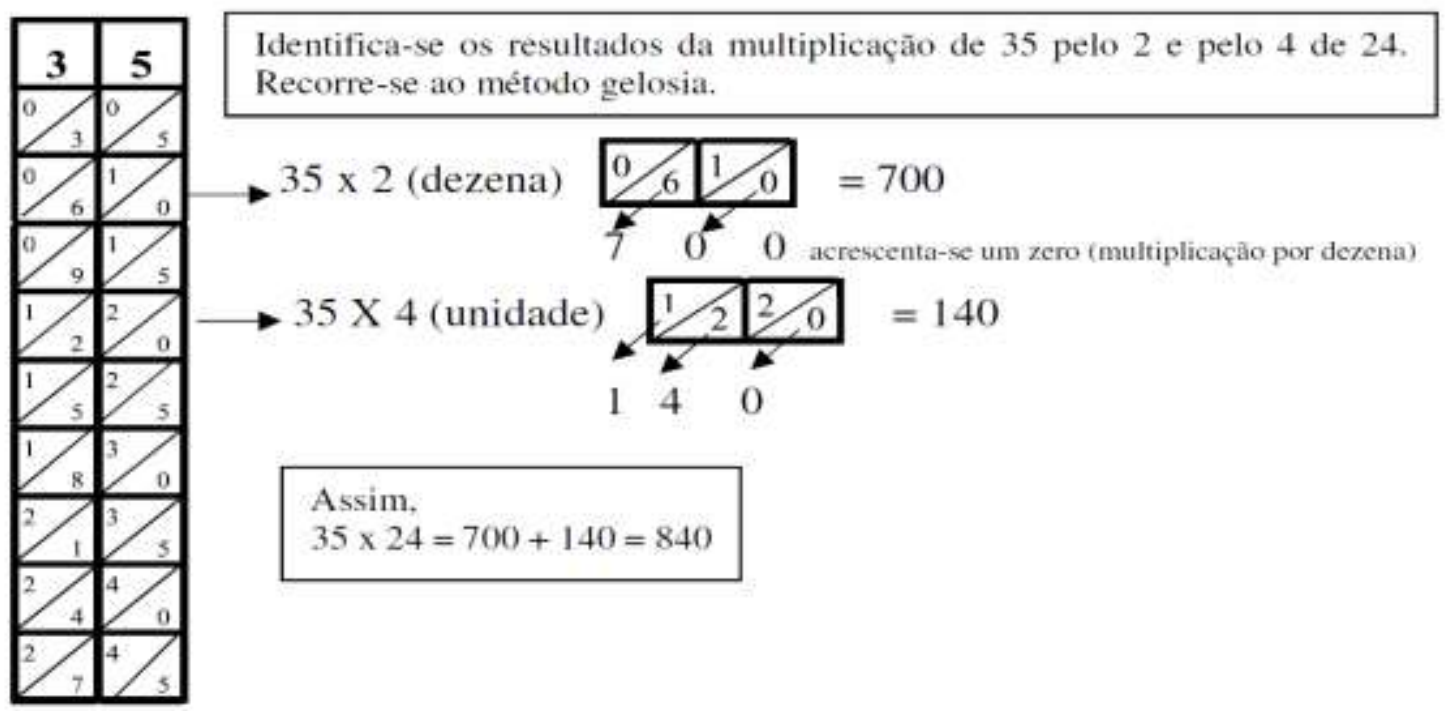


Eves (2011, p. 370), apresenta o seguinte exemplo de multiplicação realizada pelas barras de Napier $(1615$ x $365=589475)$ :

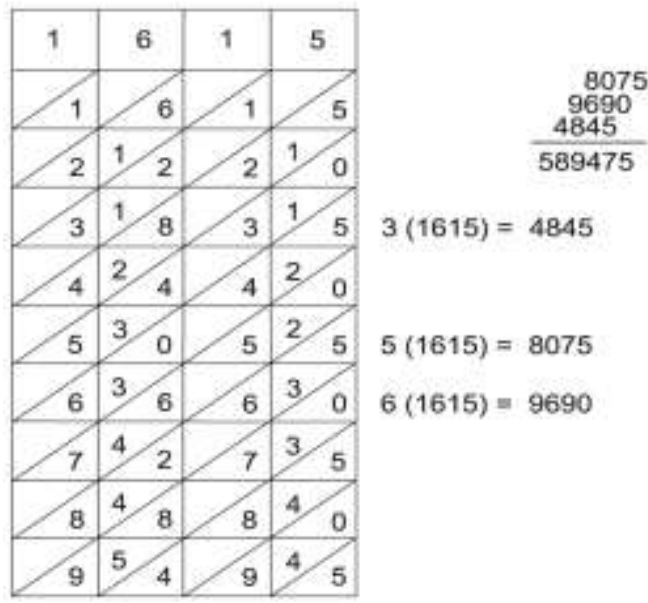

\section{Considerações finais}

No dia a dia do trabalho escolar, no desenvolvimento das ações educativas, é muito comum constatar situações que evidenciam a falta de empenho, dedicação e de envolvimento do aluno na realização das atividades pedagógicas propostas pelo professor, o que acaba contribuindo para o baixo aproveitamento e rendimento dos saberes matemáticos.

Tal fato é consequência de motivos diversos, dentre os quais, a forma como são desenvolvidas as práticas pedagógicas, que pouco motivam a efetiva participação do aluno, uma vez que são predominantemente organizadas pelos professores para conduzir o aluno ao mero treino e repetição de informações muitas vezes desconectadas da vida social e destituídas de significado.

Para o ser humano é de fato muito difícil ter o desejo, a vontade de querer aprender algo que não apresenta relação com a sua vida, com o seu contexto social, que não permite a ele verificar a importância que tem aquela informação, aquele conhecimento que está sendo ensinado. Assim sendo, essa atitude de não querer aprender conteúdos tidos como arbitrários, sem sentido, torna-se um empecilho ao desenvolvimento de uma aprendizagem significativa. 
Para buscar resolver estes problemas inerentes ao processo educativo, o professor pode lançar mão de uma variedade de recursos e métodos para ensinar Matemática. A História da Matemática, por exemplo, pode em muito, contribuir para a melhoria do ensino e da aprendizagem, motivando os alunos, enriquecendo as aulas, esclarecendo dúvidas, demonstrando a evolução dos conceitos e das ideias matemáticas, deixando claro que esta ciência está em permanente transformação, ao contrário do que muitos alunos e até mesmos professores imaginam que ela seja estática, hermética, composta de conhecimentos inquestionáveis.

Ao longo do texto ficou evidenciado que, no caso do ensino da multiplicação, o professor que atua nos primeiros anos do Ensino Fundamental poderá fazer uso na implementação da prática pedagógica de várias técnicas, procedimentos e métodos que foram desenvolvidos e utilizados no passado, com o intuito de estimular a participação ativa do estudante no processo educativo, possibilitando a ele realizar análises e comparações, verificar semelhanças e diferenças, e principalmente constatar como os saberes da Matemática são evoluções decorrentes de processos históricos.

\section{Referências}

BOYER, C. B. História da Matemática. São Paulo, SP: Ed. Edgard Blücher, 1974.

BRASIL. Secretaria de Educação Fundamental. Parâmetros Curriculares Nacionais: Matemática. Brasília, DF: MEC/SEF, 1997.

CARVALHO, D. L. Metodologia do ensino da Matemática. São Paulo, SP: Cortez, 1994.

EVES, H. Introdução à história da matemática. Campinas, SP: Editora da Unicamp, 2011.

IFRAH, G. Os números: a história de uma grande invenção. São Paulo, SP: Globo, 1992.

SOUZA, J. C. M. Matemática Divertida e Curiosa. Rio de Janeiro, RJ: Record, 2001. 